\title{
TRIM22 Activates MAPK Signaling by Regulating the Transcription of SPHK2 and Accelerating the Degradation of Raf-1 in Glioblastoma
}

\section{Xiaowei Fei}

Xijing Hospital

\section{Ya-nan Dou}

Xijing Hospital

Kai Sun

daping hospital

Jialiang Wei

Xijing Hospital

Qingdong Guo

Xijing Hospital

Li Wang

Xijing Hospital

Xiuquan Wu

Xijing Hospital

Weihao Lv

Xijing Hospital

Zhou Fei ( $\square$ feizhou123123@163.com )

Xijing Hospital

\section{Research Article}

Keywords: Glioblastoma, TRIM22, SPHK2, MAPK signaling, Proliferation

Posted Date: November 10th, 2021

DOI: https://doi.org/10.21203/rs.3.rs-1043960/v1

License: (c) (1) This work is licensed under a Creative Commons Attribution 4.0 International License.

Read Full License 


\section{Abstract \\ Background}

Tripartite motif (TRIM) 22 and mitogen-activated protein kinase (MAPK) signaling pathways play a critical role in tumor growth and therapeutic resistance of glioblastoma (GBM) respectively. However, the molecular mechanism between TRIM22 and MAPK signaling remains to be clarified.

\section{Methods}

We constructed TRIM22 knockout cell lines for molecular biology experiments, detected potential DNA fragments binding to TRIM22 by ChIP-Seq technology, and verified the sequencing results by ChIP-qPCR and CUT\&Tag technology. In addition, we constructed different TRIM22 mutants to detect the binding of proteins in MAPK signaling pathway. Finally, the therapeutic effect was verified in NOD/SCID mice. The difference between the two groups of data conforming to the normal distribution was tested by Student $t$ test.

\section{Results}

Here, we found for the first time that TRIM22 acts as a transcription factor in the nucleus and binds to exon 2 of the transcript (NM_001204160) of SPHK2 gene to regulate its expression by ChIP-Seq technology, thus indirectly affecting the downstream MAPK signaling pathway. Knockout of TRIM22 using Cas9-sgRNAs resulted in decreased mRNA level of SPHK2 in GBM cells, while overexpression of TRIM22 enhanced it. The ERK1/2 driven luciferase reporter construct identified TRIM22 as a potential activator of MAPK signaling. Knockout and overexpression of TRIM22 regulate the inhibition and activation of MAPK signaling through its RING-finger domain. Co-immunoprecipitation demonstrated that TRIM22 bound to the negative regulator Raf- 1 of MAPK signaling and accelerated its degradation by inducing K48-linked ubiquitination. The combination of the two is related to the CC domain and SPRY domain of TRIM22 and the C1D domain of Raf-1. TRIM22 also forms a complex with the downstream regulator ERK1/2 of MAPK and promotes K63-linked ubiquitination, resulting in the phosphorylation of ERK1/2. In addition, in vitro and in situ xenotransplantation models, SPHK2 inhibitor (K145), ERK1/2 inhibitor (Selumetinib) and non-phosphorylated mutant Raf-1 ${ }^{\mathrm{S} 338 \mathrm{~A}}$ inhibited the growth promoting properties of TRIM22 in GBM cell line.

\section{Conclusions}

In conclusion, our study shows that TRIM22 regulates SPHK2 transcription as a transcription factor, indirectly affects MAPK signaling, and activates MAPK signaling through post-translational modification of two critical regulators of MAPK signaling in GBM cells. 


\section{Background}

Mitogen activated protein kinase (MAPK) cascade regulates a variety of cell biological processes, such as proliferation, differentiation, apoptosis and key signaling pathways of stress response under normal and pathological conditions[1-3]. Studies have shown that MAPK is highly active in human glioblastoma (GBM) and plays a central role in many other active carcinogenic pathways[4-6]. For example, MAPK and PI3K signaling pathways cooperate to cause the pathogenesis of GBM[7]. However, the mutation or amplification of MAPK signaling subunits is rare in GBM which indicates that the abnormal activation of MAPK signaling may be due to the deregulation of pathway or oncogene.

Ubiquitination is one of the most common and important types of post-translational modification $[8,9]$. The most well studied polyubiquitin chain types include lysine 48 (K48) and lysine 63 (K63) linked. K48linked polyubiquitin chains mainly target degraded proteins, while K63-linked polyubiquitin chains regulate kinase activity, signal transduction and endocytosis[10, 11]. Post translational modifications, especially ubiquitin modifications, have become key participants in MAPK activation. For instance, LZTR1 promotes the polyubiquitination and degradation of Ras through the ubiquitin proteasome pathway, resulting in the inhibition of Ras/MAPK signaling[12].

The tripartite motif (TRIM) proteins, which regulate MAPK activity through ubiquitination, are becoming key regulators in the development of a variety of cancers, including GBM[9]. TRIM-FLMN protein TRIM45 directly interacts with RACK1 and negatively regulates PKC mediated MAPK signaling pathway[13]. GPER reduces the Bim protein through MAPK/Erk-TRIM2 signal axis and promotes the resistance of ER+ breast cancer cells to tamoxifen[14]. TRIM22 is rarely studied in most human carcinogenesis, and until now the relationship between TRIM22 and MAPK signaling pathway has not been reported. It is reported that TRIM22 regulates protein degradation and activity as an E3 ubiquitin ligase in the cytoplasm[15], but more and more studies have found that TRIM22 is mainly located in the nucleus[16]. Using ChIP-Seq, CUT\&Tag-qPCR, CRISP/Cas9 technologies, our study found that TRIM22 acts as a transcription factor in the nucleus to regulate the expression of sphingosine kinase 2 (SPHK2), and then indirectly activate the MAPK pathway downstream of SPHK2. In addition, our study found that TRIM22, as a classical E3 ubiquitin ligase, promotes K48-linked ubiquitination of Raf-1 and K63-linked ubiquitination of ERK1/2, thus directly affecting the MAPK pathway. At present, SPHK2 and MAPK inhibitors have been widely used in clinical trials and achieved good curative effects $[17,18]$. Our discovery that TRIM22 directly and indirectly regulates SPHK2/MAPK signaling pathway provides a new idea for GBM treatment.

\section{Methods}

\section{Cell culture}

Human glioma cell lines (U251MG, A172, U118, Hs683, SNB19, KNS89, H4, LN229, T98, U87MG and TJ905) and 293T cells were purchased from Genechem Co., Ltd (Shanghai, China). All cell lines were identified by short tandem repeat analysis. Primary cells P1 and P2 were taken from the Department of 
Neurosurgery of Xijing Hospital. The cells were cultured in Dulbecco's modified Eagle's medium (Gibco) containing 10\% fetal bovine serum (Gibco). Serum-free neurobasal medium containing 2\% B27 (Gibco) and $0.25 \%$ glutamine (Sigma) was used for primary cell culture. All cells were cultured in a constant temperature incubator containing $5 \%$ carbon dioxide at $37^{\circ} \mathrm{C}$.

\section{Lentivirus and plasmid transfection}

TRIM22 gene was knocked out by CRISPR/cas9 technology. The Cas9 and single-guide RNA (sgRNAs) lentiviruses were designed and constructed by Genechem Co., Ltd (Shanghai, China). The sequences of sgRNAs used are listed in Additional file 1: Table 1. Cell lines were screened with puromycin.

The mutant plasmids of TRIM22 (Flag-TRIM22- $\triangle$ RING, Flag-TRIM22- $\Delta B-B o x$, Flag-TRIM22- $\Delta$ CC and FlagTRIM22- $\triangle$ SPRY) and Raf-1 (HA-Raf-1- $\triangle$ RBD, HA-Raf-1-C1D and HA-Raf-1-CD) were designed and constructed by Hanbio Co., Ltd (Shanghai, China). Plasmid transfection was performed with jetPRIME DNA transfection reagent (Polyplus) according to the instructions of the kit.

\section{Subcellular fractionation}

Nuclear and cytoplasmic fractions from U251MG, A172, TJ905, H4, P1 and P2 were isolated using Nuclear and Cytoplasmic Extraction Kit purchased from Beyotime (Shanghai, China), according to the manufacturer's instructions. Subcellular distribution of proteins was determined using western blot analysis. GAPDH and Histone $\mathrm{H} 3$ served as loading controls for cytosolic and nuclear fractions, respectively.

\section{Cleavage Under Targets and Tagmentation (CUT\&Tag)}

CUT\&Tag was carried out according to the instructions of the following kits: Hyperactive. In-Situ ChIP Library Prep Kit for Illumina (pG-Tn5) (Cat. No.TD901-01); VAHTS DNA Clean Beads (Cat. No. N411); TruePrep Index Kit V2 for Illumina (Cat. No.TD202); Equalbit $1 \times$ dsDNA HS Assay Kit (Cat. No. EQ121). All kits were purchased from Vazyme (Nanjing, China).

\section{Quantitative real-time polymerase chain reaction (qPCR)}

qPCR was performed according to the protocol in the ChamQ SYBR Color qPCR Master Mix (Low ROX Premixed) kit (Vazyme; China). GAPDH served as the internal control. Primers sequence for qPCR are listed in Additional file 1: Table 2.

\section{Chromatin immunoprecipitation sequencing and qPCR (ChIP-Seq and ChIP-qPCR)}

ChIP-seq and ChIP-qPCR was completed with the assistance of Kangchen Biotechnology Co., Ltd (Shanghai, China). The detailed steps of the experiment are described in the Additional file 1: materials and Methods. 
Pink box represents SPHK2 gene and gray box is the 2000 BP promoter region upstream of SPHK2. Green box represents a transcript of SPHK2 NM_001243876, orange box represents another transcript NM_001204160, blue box represents the position of peak in ChIP-Seq results. The above gene sequence and transcript sequence information refer to GRCh37/hg19 genome version information, and the sequence is obtained from UCSC database (UCSC Genome Browser》 http://genome.ucsc.edu/index.html).

NM_001243876 has 6 exons and 5 introns in total, and peak is located in its intron 2 region. NM_001204160 has 6 exons and 5 introns in total. Peak is located in most of the sequences of exon 2 and part of the sequences of intron 2. According to the Peak site, NM_001204160 was selected as the study subject. Detailed site information is shown in Additional file 1: Table 3.

\section{Dual-Luciferase reporter assay}

The ERK1/2 firefly-luciferase and renilla reporter plasmids were transfected into each modified cell lines. The cells were cultured for 24 hours after plasmid transfection and the fluorescence intensity of each treatment group was detected by Dual-Luciferase Reporter Assay Kit (Promega).

\section{Immunofluorescence (IF), immunohistochemistry (IHC), Co-immunoprecipitation (Co-IP) and Western blotting (WB)}

IF, IHC and WB were performed as previously described $[19,20]$ and all antibodies used are described in Additional file 1: Table 4. To assess in vivo ubiquitination, modified cells were treated with $20 \mu \mathrm{M}$ MG132 (Apexbio; Houston, TX, USA) for 12 h before lysis, followed by western blot analysis and Co-IP."

\section{Yeast one hybrid}

Different modified potential DNA-binding proteins, the Prey, are expressed as fusions to the GAL4 activation domain in pGADT7-Rec2. The different modified target DNA sequence, or Bait Sequence, is cloned into pHIS2.1 reporter vector as tandem repeats three times. The two plasmids were transferred into Yeast strain Y187 and cultured in selected media. 3-amino-1,2,4-triazole (3-AT) is used to inhibit low levels of His3p expressed in the absence of an activating prey protein.

\section{Animal studies}

Male four-week-old NOD/SCID mice were purchased from Shenzhen Huafukang Bioscience Co., Inc., (Shenzhen, China) and randomly divided into 10 mice in each group. Different modified GBM cells expressing luciferase were implanted into the brain of mice by stereotactic injection ( $2 \mathrm{~mm}$ posterior and $1 \mathrm{~mm}$ lateral of the bregma). The tumor cells (3X106/5ul PBS) were injected into the mouse brain (depth: $2.8 \mathrm{~mm}$ ) using a micro-syringe within 2 minutes. After the operation, the skull was sealed with bone wax, and the incision was sutured and $0.5 \mathrm{ml}$ glucose was injected into the abdominal cavity. Fluorescence bioluminescence detection equipment (IVIS Lumina III) was used to detect tumor formation at 7, 15 and 
30 days after surgery. $\mathrm{K} 145(50 \mathrm{mg} / \mathrm{kg})$ and selumetinib $(50 \mathrm{mg} / \mathrm{kg})$ were given intraperitoneally every day according to the weight of mice until the end of the experiment.

\section{Statistical analysis}

All experiments were performed at least three independent biological replicates. The data are expressed as mean \pm standard deviation. Student t-test was used for statistical analysis between the two groups, and one-way ANOVA was used between multiple groups. The F-test is used to check whether the data conform to the normal distribution. SPSS for Mac software (26.0) was used for statistical analysis. $\mathrm{P}<$ 0.05 was considered statistically significant.

\section{Results}

\section{TRIM22 acts as a transcription factor to regulate the transcription of SPHK2 in the nucleus}

We collected glioma samples of different grades from Xijing Hospital for detection (NBT; $n=10$, WHO II; $n=92$, WHO III; $n=65$, WHO IV; n=70). We found that TRIM22 was highly expressed in GBM (Fig. 1a) and mainly located in the nucleus (Fig. 1b). In addition, cell lines U251MG, A172, TJ905, H4 with high expression of TRIM22 and primary GBM cell lines P1 and P2 were selected. Western blot (Fig. 1C) and immunofluorescence (Fig. 1d) showed that the expression of TRIM22 was mainly located in the nucleus. To study the role of TRIM22 in the nucleus, we used ChIP-Seq and enrichment analysis to find that TRIM22 binds to the DNA fragment of VEGF pathway related genes (MAPKAPK3, NFATC2, PP3CC, PRKCA, SPHK1, SPHK2), which may play the role of transcription factors (Fig. 1e, Additional file 1: Fig. S1a). In addition, ChIP- qPCR (Fig. 1f) in 293T and Yeast one hybrid (Fig. 1g) in vitro showed that TRIM22 could bind to the gene fragment of SPHK2. These results show that TRIM22 acts as a transcription factor to regulate the transcription of $S P H K 2$ in the nucleus.

\section{TRIM22 binds to the transcriptional region of SPHK2}

To study the detailed binding sites of TRIM22 and SPHK2, according to the information of multiple transcripts of $S P H K 2$, the transcription start site (TSS) and the position of peak in the sequencing results, four pairs of different primers were designed to explore potential binding sites (Additional file 1: S Table 3, Additional file 1: Fig. S1b). In 293T cells, ChIP-qPCR showed that TRIM22 bound most closely to the site interval of peak (Additional file 1: Fig. S1c). In addition, different TRIM22 truncates (Flag-TRIM22- $\triangle$ RING, Flag-TRIM22- $\triangle \mathrm{B}$-Box, Flag-TRIM22- $\Delta C \mathrm{C}$ and Flag-TRIM22- $\triangle$ SPRY) were transfected into U251MG, A172, TJ905, H4 and two primary cells P1 and P2 for CUT\&Tag. We were surprised to find that both TRIM22 (Fig. 2a) and its truncate (Additional file 1: Fig. S2a-b, Additional file 1: Fig. S3a-b) bind to SPHK2-4. In Yeast one hybrid, three repeated $S P H K 2-4$ were used as bait sequence, and we also obtained the results consistent with those in vivo (Fig. 2b). To further explore how TRIM22 regulates the expression of SPHK2, Using CRISPR/Cas9 technology, we constructed TRIM22-knockout (KO) cell line (Additional file 1: Fig. S1d). qPCR results showed that knockout of TRIM22 reduced the mRNA expression of SPHK2, while 
overexpression of TRIM22 could promote its expression in four cell lines and two primary cells (Fig. 2c). These results suggest that TRIM22 protein positively regulates the expression of SPHK2.

\section{TRIM22 positively regulates SPHK2/MAPK signaling pathway through K48-linked ubiquitination of Raf-1}

Our results show that TRIM22 binds transcripts of SPHK2 and positively regulates SPHK2 expression. The enrichment results of ChIP-Seq also showed that TRIM22 may affect the VEGF pathway, and although both MAKP and PI3K/AKT pathways are in the VEGF pathway, only the MAPK pathway is downstream of SPHK2. (Additional file 1: Fig. S1a). To explore the effect of TRIM22 on SPHK2/MAPK pathway, using an ERK1/2 dependent transcriptional reporter containing three copies of an ERK1/2 response element located upstream of luciferase. Dual luciferase reporter assay showed that knockout of TRIM22 inhibited the activity of MAPK signal (Fig. 3a). Knockout of TRIM22 inhibited the expression of SPHK2/MAPK pathway proteins, including SPHK2, Ras, P-Raf- ${ }^{\text {Ser338 }}$, P-MEK $1 / 2^{\text {Ser217/221 }}$ and PERK1/2 ${ }^{\mathrm{Thr} 202 / \mathrm{Tyr} 204}$. Although the expression of phosphorylated protein was down regulated after knockout of TRIM22, there was no significant change in the expression of MEK1/2 and ERK1/2 (Fig. 3b, Additional file 1: Fig. S4a). Interestingly, the expression of Raf-1 was up-regulated but the mRNA level did not change (Fig. 3c). In addition, knockout of TRIM22 attenuated the degradation of Raf-1 (Fig. 3d, Additional file 1: Fig. S4b) and reduces K48-linked ubiquitination of Raf-1 (Fig. 3e, Additional file 1: Fig. S4c). These results suggest that TRIM22 regulates SPHK2/MAPK pathway and TRIM22 deletion attenuates K48-linked ubiquitination of Raf-1.

\section{SPHK2 and MAPK inhibitors attenuated TRIM22-promoted proliferation of GBM}

It has been reported that TRIM22 is highly expressed in GBM and promotes the proliferation of GBM[21]. To explore whether TRIM22 promotes GBM proliferation through SPHK2/MAPK signaling pathway, K145, SPHK2 inhibitor and Selumetinib, a highly potent non-ATP competitive MEK1/2 inhibitor, were used for in vivo and in vitro experiments. Overexpression of TRIM22 activated MAPK signal, while the fluorescence intensity decreased significantly after treatment with K145 and Selumetinib for 24 hours (Fig. 4a, Additional file 1: Fig. S5a). K145 and Selumetinib also inhibited the growth rate of cells promoted by TRIM22 (Fig. 4b, Additional file 1: Fig. S5b) and the expression rate of Ki67 (Fig. 4c, Additional file 1: Fig. S5c). In vivo, consistent with previous reports, A172 cell line and primary cell line P1 overexpressing TRIM22 accelerated the proliferation of GBM 15 days after tumor implantation. The tumor growth rate of NOD/SCID mice injected with K145 and Selumetinib daily intraperitoneally was slower (Fig. 4d). In addition, injection of K145 and Selumetinib improved the survival rate of mice (A172: Flag-EV vs. FlagTRIM22; 44 days vs. 32.5 days; $P<0.05$; Flag-TRIM22 vs. Flag-TRIM22+K145 or FlagTRIM22+Selumetinib; 32.5 days vs. 45.5 or 49 days; $P<0.05$; P1: Flag-EV vs. Flag-TRIM22; 45.5 days vs. 35 days; $\mathrm{P}<0.05$; Flag-TRIM22 vs. Flag-TRIM22+K145 or Flag-TRIM22+Selumetinib; 35 days vs. 48 or 52 days; $\mathrm{P}<0.05$; Fig. $4 \mathrm{e})$. These results illustrate TRIM22 promotes proliferation of GBM through SPHK2/MAPK signaling pathway.

\section{TRIM22 ubiquitinates Raf-1}


TRIM22 contains four structures: RING, B-Box, CC and SPRY. To explore which structure is critical for TRIM22-mediated activation of MAPK signaling, we tested TRIM22- $\triangle \mathrm{RING}$, TRIM22- $\Delta \mathrm{B}-\mathrm{B}$ ox, TRIM22- $\triangle \mathrm{CC}$ and TRIM22- $\triangle$ SPRY in ERK1/2 luciferase reporter assays compared with TRIM22-FL and EV groups. The results showed that compared with TRIM22-FL group, only TRIM22- $\triangle$ RING group significantly reduced luciferase activity (Fig. 5a, Additional file 1: Fig. S6a). Furthermore, overexpression of TRIM22 increased the expression of key proteins in SPHK2/MAPK pathway, while the lack of RING domain inhibited this change. The change trend of Raf- 1 protein was opposite to that of other proteins (Fig. 5b, Additional file 1: Fig. S6b). In CHX assay, overexpression of TRIM22 accelerated the degradation of Raf-1 and the results were similar in TRIM22- $\triangle$ RING group and Flag-EV group (Fig. 5c, Additional file 1: Fig. S6c). Both in vitro (Fig. 5d) and in vivo (Fig. 5e, Additional file 1: Fig. S6d), overexpression of TRIM22 induced endogenous K48-linked ubiquitination of Raf-1, while the RING domain deletion had no effect. These results illustrate that the change of Raf- 1 is caused by ubiquitin degradation pathway induced by TRIM22.

To investigate whether TRIM22 binds directly to Raf-1. We performed Co-IP experiments using 293T cells transfected with Flag-TRIM22 and HA-Raf-1 and the results show that Flag and HA bring down each other (Fig. 5f). Furthermore, the physical binding of endogenous TRIM22 and Raf-1 was also confirmed in four cell lines and two primary cells (Fig. 5g, Additional file 1: Fig. S6e). To further study which domain of the two proteins plays a crucial role in binding, a series of deletion mutants of TRIM22 and Raf- 1 were constructed and transfected into 293T cells. The CC and SPRY domains of TRIM22 are the necessary conditions for bringing down HA-Raf-1, while the C1d domain of Raf-1 is the necessary condition for bringing down f Flag-TRIM22 (Fig. 5h). Thus, TRIM22 promotes proteasomal-mediated degradation of Raf-1 via its RING domain, a negative regulator of MAPK signaling.

\section{Raf-1 mediates the proliferation of GBM promoted by TRIM22}

Phosphorylation at S338 site activates Raf-1 protein[22]. To further study the role of Raf-1 in TRIM22 promoted proliferation of GBM, we constructed a mutant of phosphorylated Raf-1, and the serine at site 338 was mutated to alanine (Raf- ${ }^{\mathrm{S} 338 \mathrm{~A}}$ ). The plasmid was packaged with lentivirus and transfected into cells for stable expression. Luciferase Report showed that Raf-1 ${ }^{\mathrm{S} 338 \mathrm{~A}}$ inhibited the activation of MAPK pathway caused by overexpression of TRIM22 (Fig. 6a, Additional file 1: Fig. S7a). The growth of GBM cells (Fig. 6b, Additional file 1: Fig. S7b) and positive rate of Ki67 (Fig. 6c, Additional file 1: Fig. S7c) promoted by overexpression of TRIM22 was also inhibited by Raf- ${ }^{\mathrm{S} 338 \mathrm{~A}}$. Moreover, in vivo, compared with Flag-TRIM22 group, Raf-1 ${ }^{\text {S338A }}$ group showed significant inhibitory effect at 15 days after intracranial tumor implantation (Fig. 6d). The Flag-TRIM22 group had shorter survival, while there was no difference between Flag-EV and Raf-1 ${ }^{\text {S338A }}$ group (A172; Flag-EV vs. Flag-TRIM22+ Raf-1 ${ }^{\text {S338A; }} 41$ days

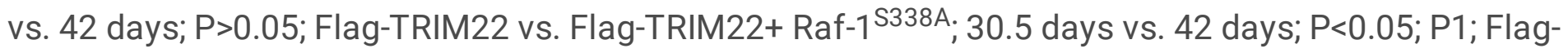
EV vs. Flag-TRIM22+ Raf-1 ${ }^{\text {S338A }} 47$ days vs. 42 days; $P>0.05$; Flag-TRIM22 vs. Flag-TRIM22+ Raf-1 5338 ; 29.5 days vs. 42 days; $P<0.05$; Fig. 6 e). These results indicated that Raf- 1 is a key effector in TRIM22promoted growth of GBM cell populations in vitro and in vivo. 


\section{TRIM22 promotes K63-linked ubiquitination of ERK1/2}

TRIM22 knockout decreased the expression of key proteins of SPHK2/MAPK pathway, including SPHK2, Ras and phosphorylated MEK1/2 and ERK1/2 (Fig. 3b, Additional file 1: Fig. S4a), while overexpression of TRIM22 promoted its up regulation (Fig. 5b, Additional file 1: Fig. S6b). The parallel change trend of these proteins and TRIM22 makes us speculate whether TRIM22 may directly regulate the activation of these proteins. To explore whether TRIM22 directly regulates these key proteins, anti-TRIM22 antibody was used for Co-IP experiment. We found that ERK1/2 was associated with TRIM22 in four GBM cell lines and two primary GBM cells (Fig. 7a, Additional file 1: Fig. S8a). The same results were obtained by Co-IP with anti-ERK1/2 antibody (Fig. 7b, Additional file 1: Fig. S8b). Furthermore, we found that K63 ubiquitination levels of ERK1/2 paralleled TRIM22 protein levels. TRIM22 knockout reduced K63-linked ubiquitination levels of ERK1/2 (Fig. 7c, Additional file 1: Fig. S8c) and overexpression TRIM22 promotes it. The specific mechanism may also be related to the RING domain of TRIM22(Fig. 7d, Additional file 1: Fig. S8d). These results suggested that TRIM22 might regulate activation of MAPK through ERK1/2 activation.

\section{Discussion}

TRIM22 plays a key role as E3 ubiquitin ligase in tumors. Our study found for the first time that TRIM22 regulates SPHK2 gene transcription as a transcription factor in the nucleus. We found that the main binding gene sites of TRIM22 are located in intron 2 of NM_001243876 transcript and exon 2 of NM_001204160 transcript. In addition, it is found that TRIM22 positively regulates the expression of SPHK2 gene and indirectly affects the activation of MAPK signaling. TRIM22 also directly binds to MAPK pathway core proteins Raf-1 and ERK1/2, regulates degradation of Raf-1 through K48-linked ubiquitination, and regulates activation of ERK1/2 by K63-linked ubiquitination. These results illustrate that TRIM22 directly or indirectly regulates MAPK signaling, thereby promoting GBM proliferation (Fig. 7e).

TRIM22 contains four domains, RING domain, B-Box domain, CC domain and SPRY domain. It has been reported that the RING domain is involved in the function of ubiquitination as a classical domain of the TRIM family[15]. The specificity of the Ub-binding system arises from the direct binding of E3 ligases to their substrates. Our findings that TRIM22 binds Raf- 1 and ERK1/2 and promotes K48-linked and K63linked ubiquitination respectively, are consistent with the functions reported in the literature. In addition, we found that deletion of the RING domain significantly inhibited SPHK2/MAPK signaling activation by overexpressing TRIM22, suggesting that the RING domain of TRIM22 may regulate signaling activation in addition to its role as an E3 ubiquitin ligase. Although the RING domain plays a functionally important role and has also been shown to be the main domain promoting proliferation in GBM, the binding of TRIM22 to Raf-1 does not appear to be related to the RING domain. Studies have reported that TRIM22 binds IKBa associated with its B-Box domain, CC domain and SPRY domain[21], while our study found that the binding of TRIM22 and Raf- 1 is associated with the CC domain and SPRY domain. On the other hand, we also found that TRIM22 is independent of its four domains when it acts as a transcription factor to regulate SPHK2 transcription. Polymorphisms in the function of this domain of TRIM22 make it 
necessary to further investigate which region of TRIM22 may be associated with the development of human gliomas.

Raf-1 is a major effector recruited through the GTP-binding protein Ras and activates the MEK-MAP kinase pathway[23]. Activation of Raf-1 involves phosphorylation of multiple activation sites including

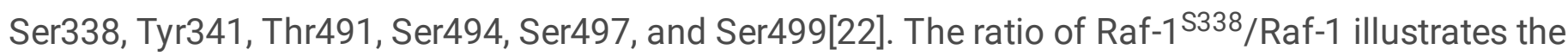
activation of Raf-1, and our study found that the synergistic effect of TRIM22 on both Raf-1 phosphorylation and degradation prompted Raf activation. In addition, the p44/42 MAPK (ERK1/2) signaling pathway is activated in response to a variety of extracellular stimuli. MEK1 and MEK2 activate p44 and p42 by phosphorylating the activation loop residues Thr202/Tyr204 and Thr185/Tyr187, respectively[24, 25]. It is believed that ERK1/2 is a key target for cancer diagnosis and treatment[26]. We demonstrate that TRIM22 targets Raf- 1 for proteasome-mediated degradation and further enhances MAPK signaling activation by modifying K63-linked polyubiquitin chains on ERK1/2. However, in addition to $\mathrm{K} 48$ and $\mathrm{K} 63, \mathrm{~K} 6, \mathrm{~K} 11, \mathrm{~K} 27, \mathrm{~K} 29$ and $\mathrm{K} 33$-linked polyubiquitin chains also play important roles in the regulation of Raf-1 and ERK1/2, but whether TRIM22 regulates ubiquitination and other phosphorylation sites at these sites of Raf- 1 and ERK $1 / 2$ also needs further study.

K145 is a selective inhibitor of SPHK2 and shows excellent antitumor activity in vivo and in vitro[27, 28]. Liu's group[29] found that K145 can act as a dual pathway inhibitor and inhibit both ART and AKT signaling pathway phosphorylation while inhibiting SPHK2, thereby achieving anti-proliferative and apoptotic effects in leukemia U937 cells. Selumetinib is a highly potent and selective, non-ATPcompetitive MEK1/2 inhibitor[30], and the results from National Cancer Institute showed a good therapeutic effect [31] in a clinical trial of Selumetinib in the treatment of low-grade astroglioma and metastatic low-grade glioma (NCT04166409) conducted on January 3, 2020. Our study found that K145 and Selumetinib could significantly inhibit the proliferation of GBM promoted by overexpression of TRIM22 both in vivo and in vitro.

\section{Conclusion}

In conclusion, our study reveals for the first time an entirely new regulatory mechanism of MAPK activation, finding that TRIM22, which is highly expressed in GBM, regulates the activation of MAPK signaling by both direct (E3 ubiquitin ligase) and indirect (transcription factor) means. TRIM22 induces SPHK2/MAPK signaling activation in GBM, driving tumor growth and progression. Finally, our study defines TRIM22 as a candidate therapeutic target. Drug inhibition of its E3 ligase activity or TRIM22/SPHK2/MAPK interaction may provide a promising strategy for treating GBM.

\section{Abbreviations}

TRIM22: Tripartite motif (TRIM) 22; MAPK: mitogen-activated protein kinase; GBM: glioblastoma; SPHK2: sphingosine kinase 2 . 


\section{Declarations}

\section{Ethics Approval and Consent to Participate}

All primary glioma tissue samples (WHO II; $n=92$, WHO III; $n=65$, WHO IV; $n=70$ ) and nonneoplastic brain tissue samples (NBT; $n=10$ ) were obtained from the Department of Neurosurgery at Xijing Hospital. Ethical approval was obtained from Xijing Hospital Research Ethics Committee, and written informed consent was obtained from each patient. All experimental procedures were approved by the Institutional Animal Care and Use Committee of Fourth Military Medical University. The study was performed in accordance with the Declaration of Helsinki.

\section{Consent for publication}

Not applicable.

\section{Availability of data and materials}

The datasets used and/or analysed during the current study are available from the corresponding author on reasonable request.

\section{Competing interests}

The authors declare that they have no competing interests

\section{Funding}

The work was supported by National Natural Science Foundation of China (Grant No. 81872049).

\section{Author's contributions}

FXW, DYN and SK designed the study, performed the experiments, and prepared the manuscript, and they contributed equally to this work. WJL, GQD, WL, WXQ and LWH were involved in experiment performance and data collection. FXW had written the original draft. FZ were responsible for the supervision of the entire project and were involved in the study design, data interpretation and manuscript preparation. All authors read and approved the final manuscript.

\section{Acknowledgements}

Not applicable.

\section{References}

1. Baccarini M. Second nature: Biological functions of the Raf-1 "kinase". FEBS Lett. 2005;579(15):03277. 
2. Meloche S, Pouysségur J. The ERK1|[sol]|2 mitogen-activated protein kinase pathway as a master regulator of the G1- to S-phase transition. Oncogene. 2007;26(22):3227.

3. Roux PP, Blenis J. ERK and p38 MAPK-activated protein kinases: a family of protein kinases with diverse biological functions. Microbiol Mol Biol Rev. 2004;68(2):320-44.

4. Daniel P, Filiz G, Mantamadiotis T. Sensitivity of GBM cells to cAMP agonist-mediated apoptosis correlates with CD44 expression and agonist resistance with MAPK signaling. Cell Death Dis. 2016;7(12):e2494.

5. Chen X, Hao A, Li X, Ye K, Zhao C, Yang H, et al. Activation of JNK and p38 MAPK Mediated by ZDHHC17 Drives Glioblastoma Multiforme Development and Malignant Progression. Theranostics. 2020;10(3):998-1015.

6. Xu Y, Sun Q, Yuan F, Dong H, Zhang H, Geng R, et al. RND2 attenuates apoptosis and autophagy in glioblastoma cells by targeting the p38 MAPK signalling pathway. Journal of experimental \& clinical cancer research : CR. 2020;39(1):174.

7. Vitucci M, Karpinich N, Bash R, Werneke A, Schmid R, White K, et al. Cooperativity between MAPK and PI3K signaling activation is required for glioblastoma pathogenesis. Neuro Oncol. 2013;15(10):131729.

8. Shi D, Grossman S. Ubiquitin becomes ubiquitous in cancer: emerging roles of ubiquitin ligases and deubiquitinases in tumorigenesis and as therapeutic targets. Cancer Biol Ther. 2010;10(8):737-47.

9. Hatakeyama S. TRIM proteins and cancer. Nature reviews Cancer. 2011;11(11):792-804.

10. Akutsu M, Dikic I, Bremm A. Ubiquitin chain diversity at a glance. J Cell Sci. 2016:jcs.183954.

11. Swatek KN, Komander D. Ubiquitin modifications. Cell Res. 2016.

12. Bigenzahn J, Collu G, Kartnig F, Pieraks M, Vladimer G, Heinz L, et al. LZTR1 is a regulator of RAS ubiquitination and signaling. Science (New York, NY). 2018;362(6419):1171-7.

13. Sato T, Takahashi H, Hatakeyama S, Iguchi A, Ariga T. The TRIM-FLMN protein TRIM45 directly interacts with RACK1 and negatively regulates PKC-mediated signaling pathway. Oncogene. 2015;34(10):1280-91.

14. Yin H, Zhu Q, Liu M, Tu G, Li Q, Yuan J, et al. GPER promotes tamoxifen-resistance in ER+ breast cancer cells by reduced Bim proteins through MAPK/Erk-TRIM2 signaling axis. Int J Oncol. 2017;51(4):1191-8.

15. Duan Z, Gao B, Wei X, Xiong S. Identification of TRIM22 as a RING finger E3 ubiquitin ligase. Biochem Biophys Res Commun. 2008;374(3):502-6.

16. Bo G, Duan Z, Wei X, Xiong S. Tripartite motif-containing 22 inhibits the activity of hepatitis B virus core promoter, which is dependent on nuclear-located RING domain. Hepatology. 2010;50(2).

17. Zhang F, Hu W, Qu L, Cang C. Sphingosine kinase 2 inhibitor ABC294640 suppresses neuronal excitability and inhibits multiple endogenously and exogenously expressed voltage-gated ion channels in cultured cells. Channels (Austin, Tex). 2020;14(1):216-30. 
18. Dymond A, So K, Martin P, Huang Y, Severin P, Mathews D, et al. Effects of cytochrome P450 (CYP3A4 and CYP2C19) inhibition and induction on the exposure of selumetinib, a MEK1/2 inhibitor, in healthy subjects: results from two clinical trials. Eur J Clin Pharmacol. 2017;73(2):175-84.

19. Fei X, Wang J, Chen C, Ding B, Fu X, Chen W, et al. Eupatilin inhibits glioma proliferation, migration, and invasion by arresting cell cycle at G1/S phase and disrupting the cytoskeletal structure. Cancer Manag Res. 2019;11:4781-96.

20. Fei X, He Y, Chen J, Man W, Chen C, Sun K, et al. The role of Toll-like receptor 4 in apoptosis of brain tissue after induction of intracerebral hemorrhage. J Neuroinflammation. 2019;16(1):234.

21. Ji J, Ding K, Luo T, Zhang X, Chen A, Zhang D, et al. TRIM22 activates NF-KB signaling in glioblastoma by accelerating the degradation of IKBa. Cell Death Differ.

22. Chong H, Lee J, Guan K. Positive and negative regulation of Raf kinase activity and function by phosphorylation. The EMBO journal. 2001;20(14):3716-27.

23. Avruch J, Zhang X, Kyriakis J. Raf meets Ras: completing the framework of a signal transduction pathway. Trends Biochem Sci. 1994;19(7):279-83.

24. Rubinfeld H, Seger R. The ERK cascade: a prototype of MAPK signaling. Mol Biotechnol. 2005;31(2):151-74.

25. Murphy LO, Blenis J. MAPK signal specificity: the right place at the right time. Trends Biochem Sci. 2006;31(5):268-75.

26. Roberts P, Der C. Targeting the Raf-MEK-ERK mitogen-activated protein kinase cascade for the treatment of cancer. Oncogene. 2007;26(22):3291-310.

27. LeBlanc F, Pearson J, Tan S, Cheon H, Xing J, Dunton W, et al. Sphingosine kinase-2 is overexpressed in large granular lymphocyte leukaemia and promotes survival through $\mathrm{Mcl}-1$. $\mathrm{Br} \mathrm{J}$ Haematol. 2020;190(3):405-17.

28. Zhang R, Li L, Yuan L, Zhao M. Hypoxic preconditioning protects cardiomyocytes against hypoxia/reoxygenation-induced cell apoptosis via sphingosine kinase 2 and FAK/AKT pathway. Exp Mol Pathol. 2016;100(1):51-8.

29. Liu K, Guo T, Hait N, Allegood J, Parikh H, Xu W, et al. Biological characterization of 3-(2-aminoethyl)-5-[3-(4-butoxyl-phenyl)-propylidene]-thiazolidine-2,4-dione (K145) as a selective sphingosine kinase-2 inhibitor and anticancer agent. PLoS One. 2013;8(2):e56471.

30. Dymond A, Elks C, Martin P, Carlile D, Mariani G, Lovick S, et al. Pharmacokinetics and pharmacogenetics of the MEK1/2 inhibitor, selumetinib, in Asian and Western healthy subjects: a pooled analysis. Eur J Clin Pharmacol. 2017;73(6):717-26.

31. MEK1/2 Inhibition Is Effective in Subsets of Pediatric Low-Grade Glioma. Cancer Discov. 2019;9(8):OF11.

\section{Figures}



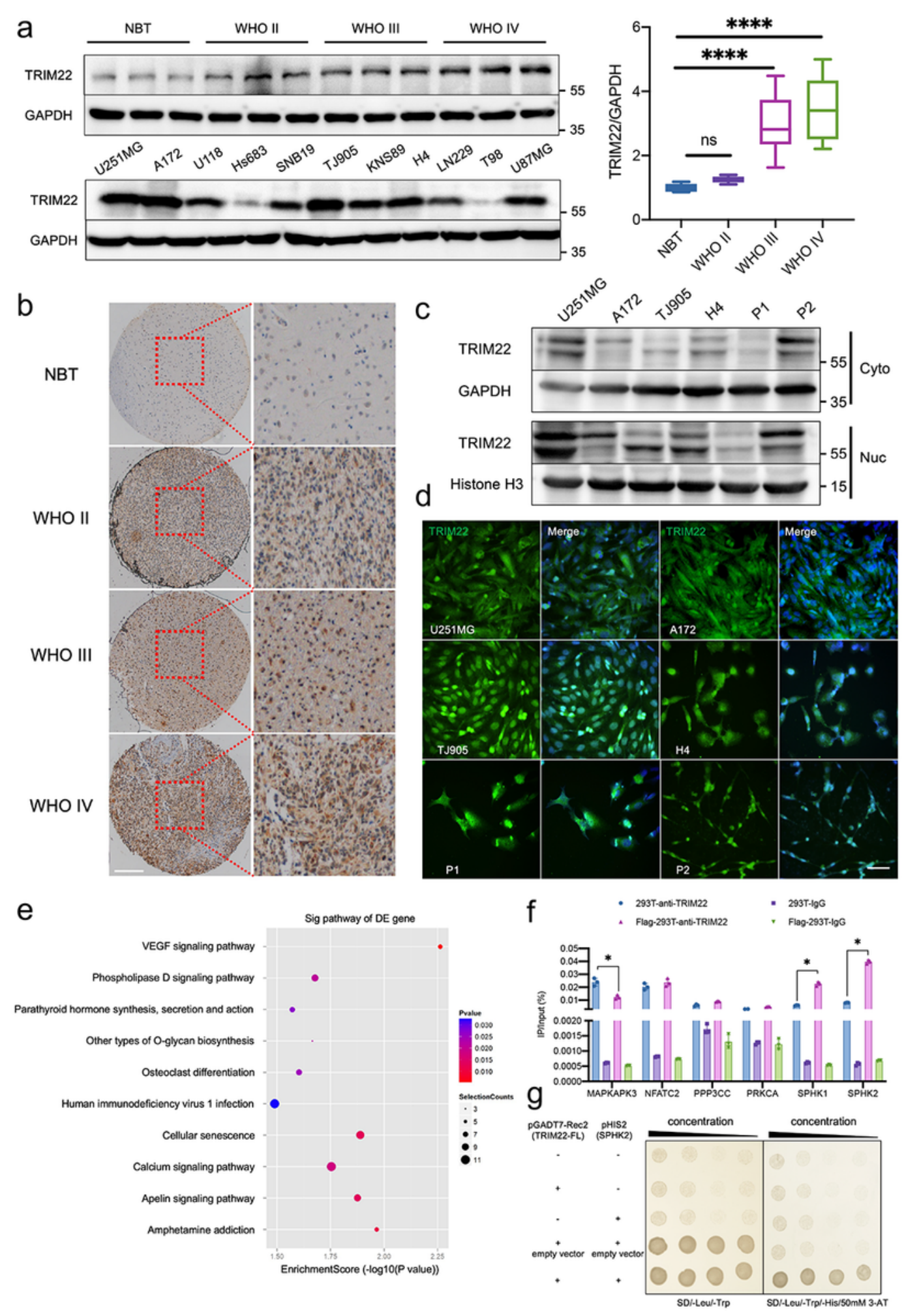

\section{Figure 1}

TRIM22 regulates transcription of SPHK2 as a transcription factor in the nucleus. a The Representative Western blot images and quantification of TRIM22 expression in different grades of gliomas (upper) and TRIM22 expression of 11 different GBM cell lines was detected by Western blot (lower). b The localization of TRIM22 in clinical specimens was detected by immunohistochemistry. Scale bar: $200 \mu \mathrm{m}$. Number of clinical samples in a and b: NBT; $n=10$, WHO II; n=92, WHO III; n=65, WHO IV; n=70. c Expression of 
TRIM22 in cytoplasm and nucleus of U251MG, A172, TJ905, H4 and primary GBM cells P1 and P2. d Localization of TRIM22 in six cells. Scale bar: $50 \mu \mathrm{m}$. e After 293T cells were transfected with FlagTRIM22 overexpression plasmid, ChIP-Seq was carried out to analyze the differential genes. $f$ ChIP-qPCR experiment was used to verify the results of ChIP-Seq. $g$ Yeast one hybrid experiment verified the binding of TRIM22 and SPHK2 in vitro. (All data represent mean \pm SEM $n \geq 3$ ). Student's t test: ${ }^{*} P<0.05$.

a
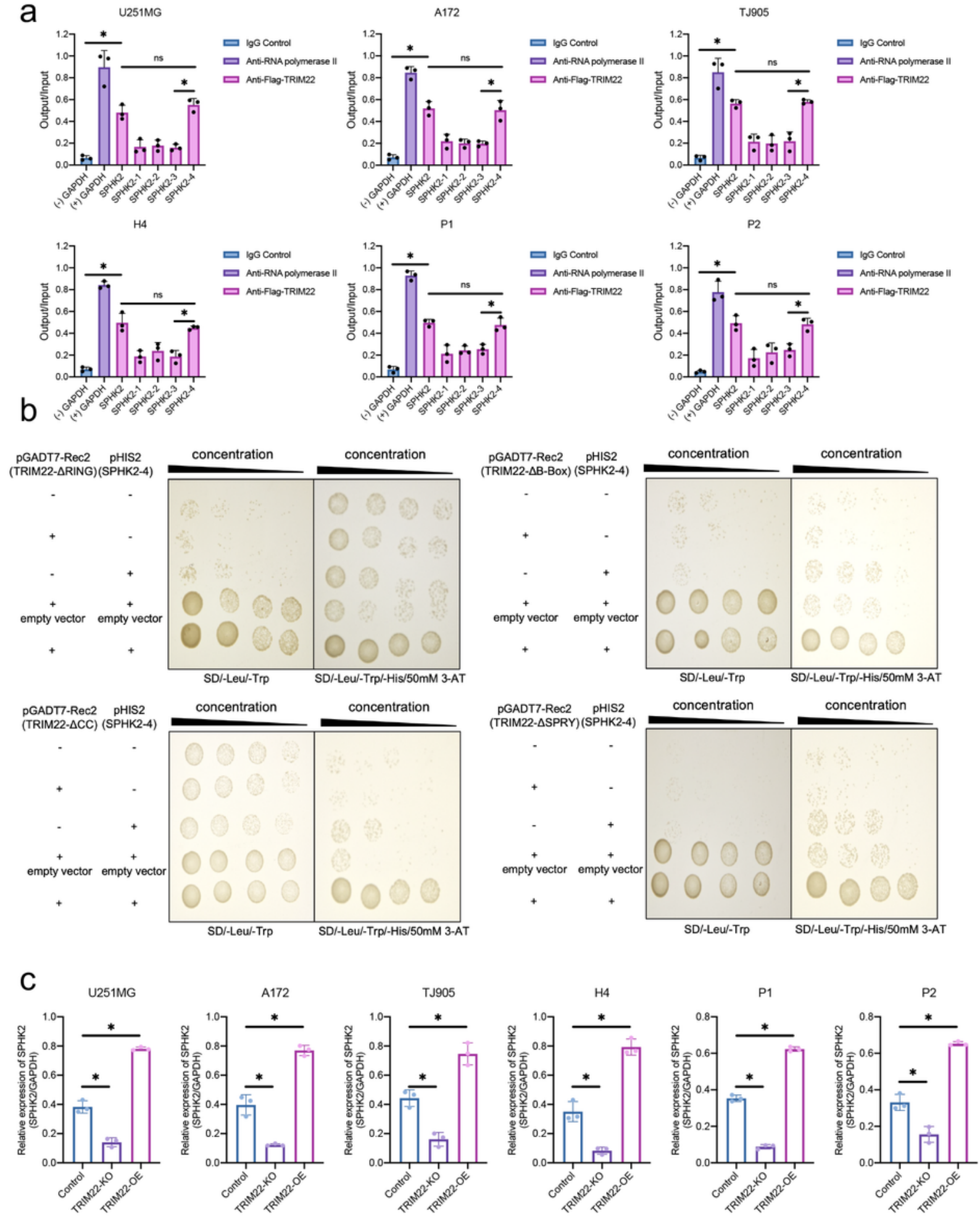

Figure 2 
TRIM22 binds to the transcriptional region of SPHK2 and positively regulates its transcription. a In U251MG, A172, TJ905, H4 and primary GBM cells P1 and P2, different binding sites of TRIM22 and SPHK2 (1-4) were detected by CUT\&Tag. $b$ In vitro yeast one hybrid was used to detect the binding of different TRIM22 truncated mutants (Flag-TRIM22- $\triangle$ RING, Flag-TRIM22- $\triangle \mathrm{B}-\mathrm{Box}$, Flag-TRIM22- $\triangle \mathrm{CC}$ and Flag-TRIM22- $\triangle$ SPRY) to SPHK2-4. c Effect of knockout or overexpression of TRIM22 on mRNA of SPHK2. (All data represent mean \pm SEM $n \geq 3$ ). Student's $t$ test: ${ }^{*} P<0.05$.

\section{a}
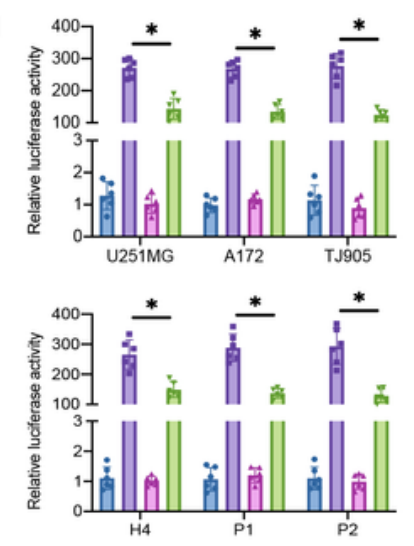

C

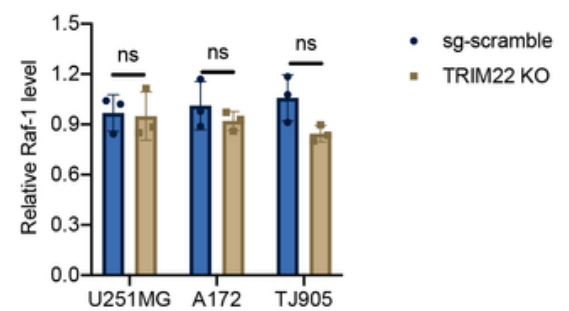

d
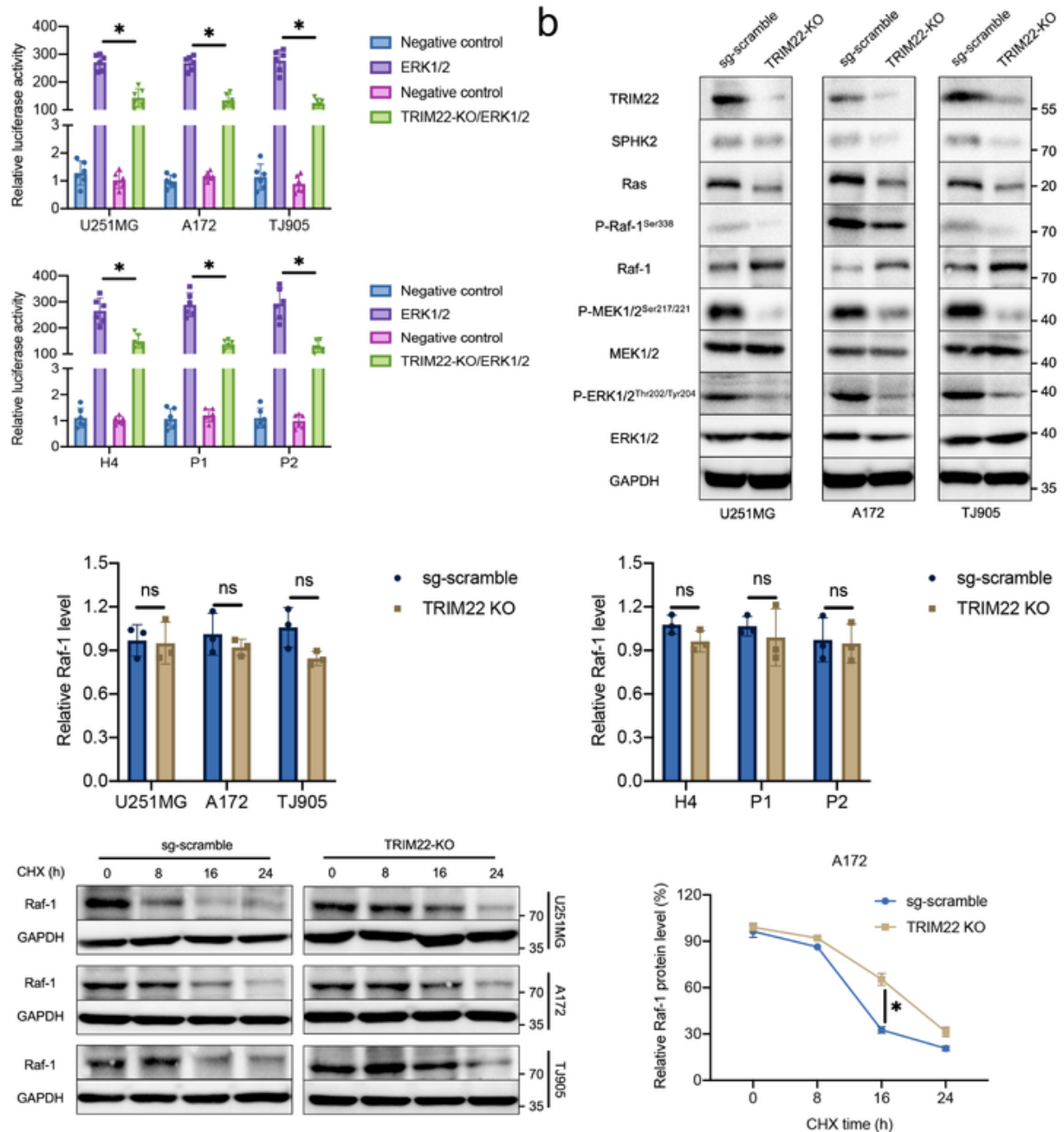

U251MG
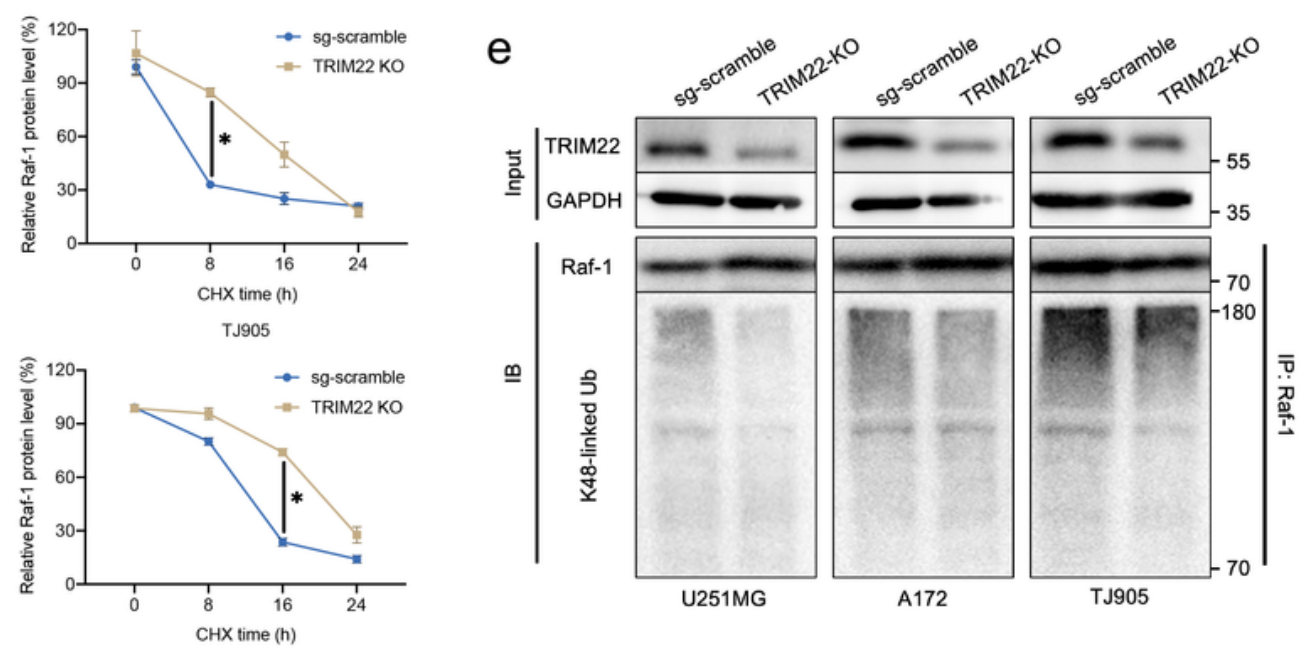

Figure 3 
TRIM22 delete inhibit SPHK2/MAPK signaling via K48-linked ubiquitination of Raf-1. a Luciferase activity from U251MG, A172, TJ905, H4 and primary GBM cells P1 and P2 transfected with sgRNA-TRIM22, along with a reporter plasmid carrying the ERK1/2 promoter relative to negative control. b The expression of SPHK2/MAPK pathway core protein was detected by Western blot. c The mRNA level of Raf- 1 was detected by qPCR. $d$ Western blot analysis of Raf-1 protein in TRIM22-KO cells treated with cycloheximide ( $\mathrm{CHX} ; 25 \mu \mathrm{g} / \mathrm{mL}$ ) for $0,8,16$, and $24 \mathrm{~h}$. e IP experiment was used to detect the endogenous K48-linked ubiquitination of Raf-1 after TRIM22 knockout. (All data represent mean $\pm S E M n \geq 3$ ). Student's $t$ test: *P $<0.05$. 

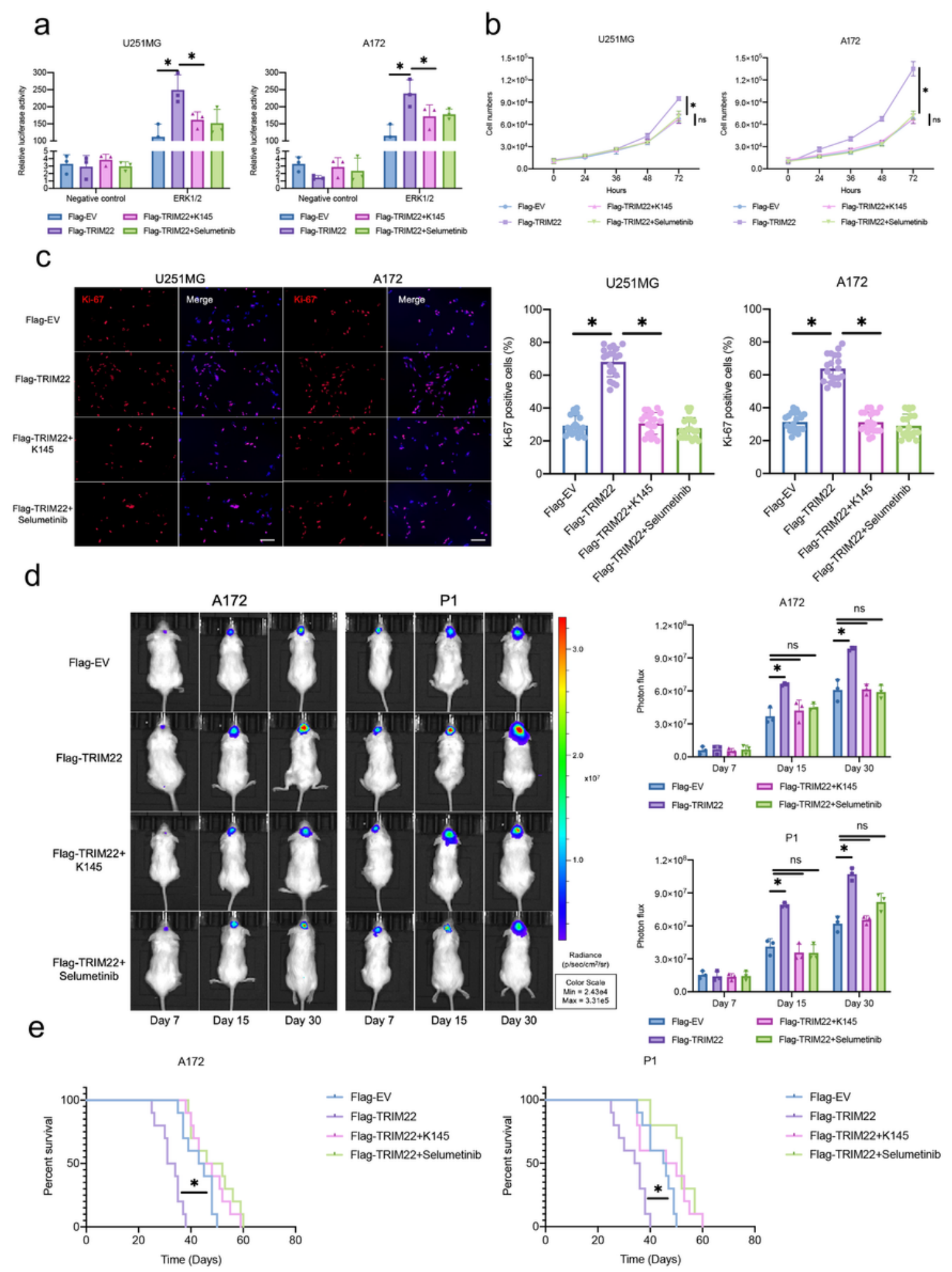

- Flag-EV

- Flag-TRIM22

- Flag-TRIM22+K145

- Flag-TRIM22+Selumetinib

Figure 4

SPHK2/MAPK pathway regulates the proliferation of GBM in vivo and in vitro. a Luciferase activity from U251MG and A172 treated with K145 and Selumetinib, along with a reporter plasmid carrying the ERK $1 / 2$ promoter relative to negative control. b Growth curves generated using cell counting over $72 \mathrm{~h}$ for the cells indicated. c Representative images and quantification of Ki-67 immunofluorescence staining from

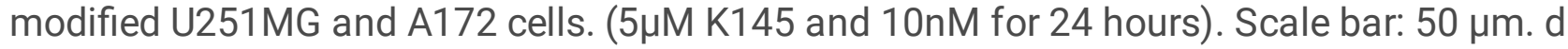


Representative images and quantification of in vivo imaging. e Kaplan-Meier survival analysis and Logrank test performed with survival data from indicated groups. (All data represent mean $\pm S E M n \geq 3$ ). Student's t test: ${ }^{*} \mathrm{P}<0.05$.

a

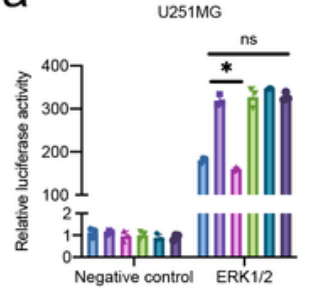

$\square$ Flag-EV

$\square$ Flag-TRIM22-FL

$\square$ Flag-TRIM22- $\triangle$ RING

$\square$ Flag-TRIM22- $\triangle \mathrm{B}-\mathrm{B}$ ox

口 Flag-TRIM22- $\triangle \mathrm{CC}$

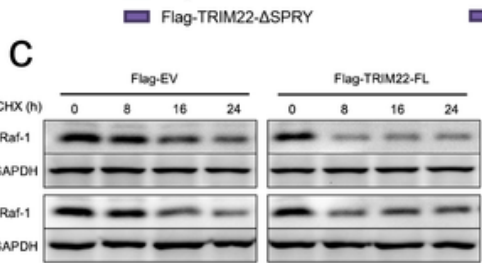
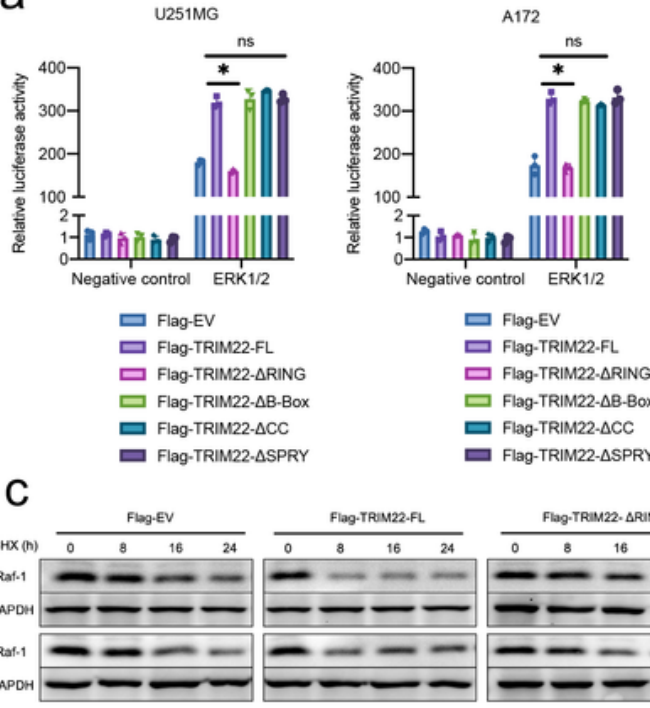

$\square$ Flag-EV

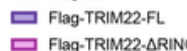

口 Flag-TRIM22 -18 Box

口 Flag-TRIM22- $\triangle \mathrm{CC}$

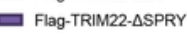

$\mathrm{b}$
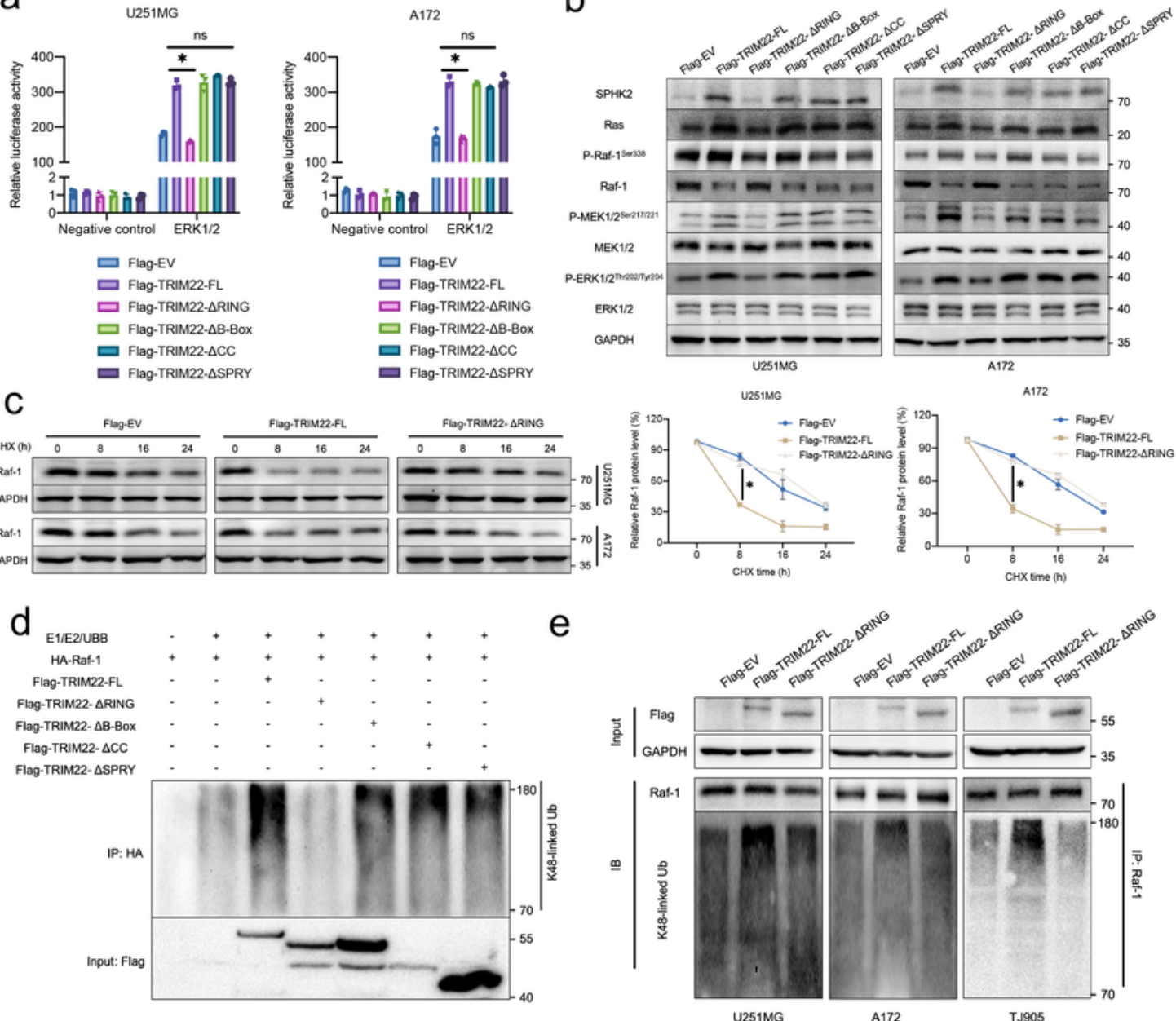

f
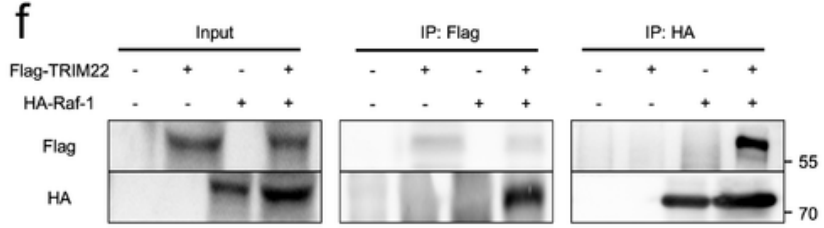

g

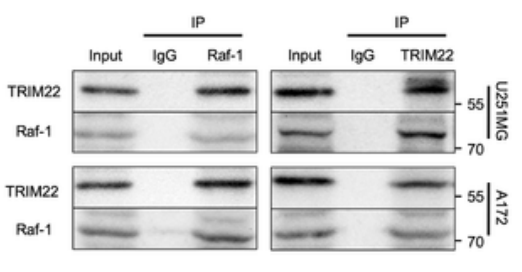

$\mathrm{h}$

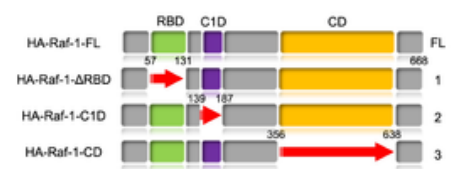

Flag-TRIM22-FL RING B-80x coled-coil SPRY FL Flag-TRIM22. $\Delta R I N G$ Flag-TRIM22-AB-Box $\square \square \square \Rightarrow \square$ IP: Flag Flag-TRIM22-ACC $\square \square \square \square$ Flag-TRIM22- $\triangle$ SPRY
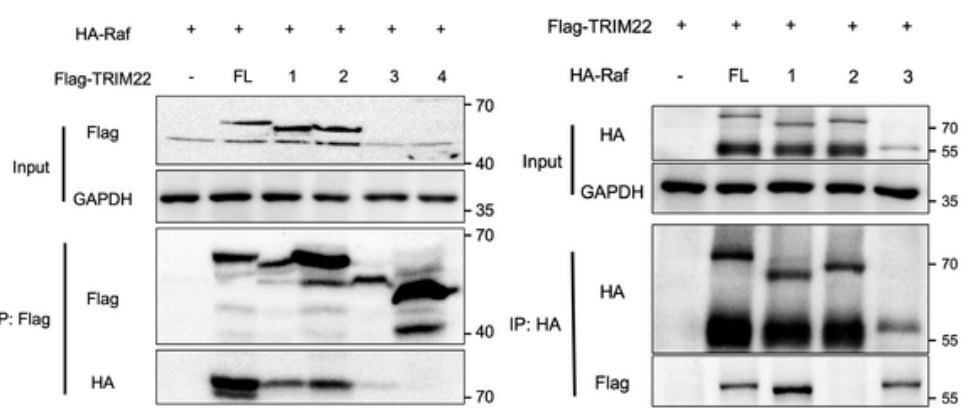

\section{Figure 5}

TRIM22 binds to Raf- 1 and regulates SPHK2/MAPK signaling through its RING domain. a Luciferase activity from U251 MG and A172 transfected with different TRIM22 truncation mutants. b The effects of 
different TRIM22 truncation mutants on the core protein of SPHK2/MAPK pathway were detected by Western blot in U251MG and A172. c Western blot analysis of Raf-1 protein in different modified cells treated with cycloheximide (CHX;25 $\mu \mathrm{g} / \mathrm{mL}$ ) for $0,8,16$, and $24 \mathrm{~h}$. d ubiquitination of Raf-1 in an in vitro assay. e In vivo ubiquitination assay of Raf-1 in modified U251 MG, A172 and TJ905. f Co-IP was used to detect the exogenous binding of TRIM22 and Raf-1 in 293T cells transfected with Flag-TRIM22 and HARaf-1. g exogenous binding of TRIM22 and Raf-1 in U251MG and A172 using anti-TRIM22 and anti-Raf-1 antibodies. h Schematic representation of wild-type TRIM22 and Raf-1 and the indicated deletion mutants. Western blot analysis of Co-IPs in 293T cells transfected with Flag-TRIM22/HA-Raf-1 alone or together with indicated HA-Raf-1/ Flag-TRIM22 constructs. (All data represent mean $\pm S E M n \geq 3$ ). Student's t test: ${ }^{*} \mathrm{P}<0.05$. 


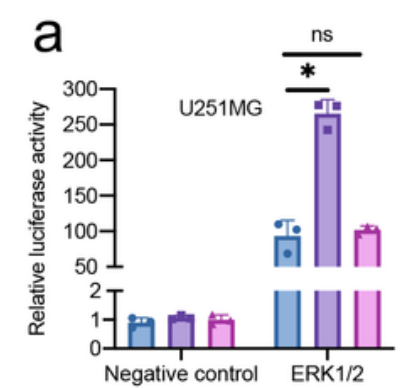

$\square$ Flag-EV

$\square$ Flag-TRIM22

$\square$ Flag-TRIM22+Raf-1 ${ }^{\text {S338A }}$

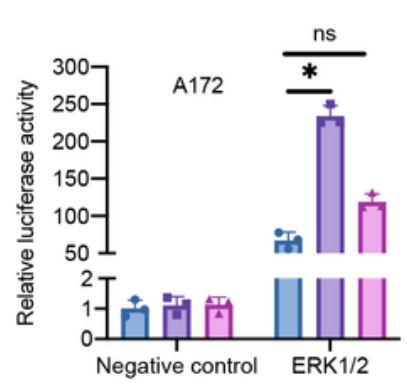

$\square$ Flag-EV

Flag-TRIM22

$\square$ Flag-TRIM22+Raf-1 ${ }^{\text {S338A }}$
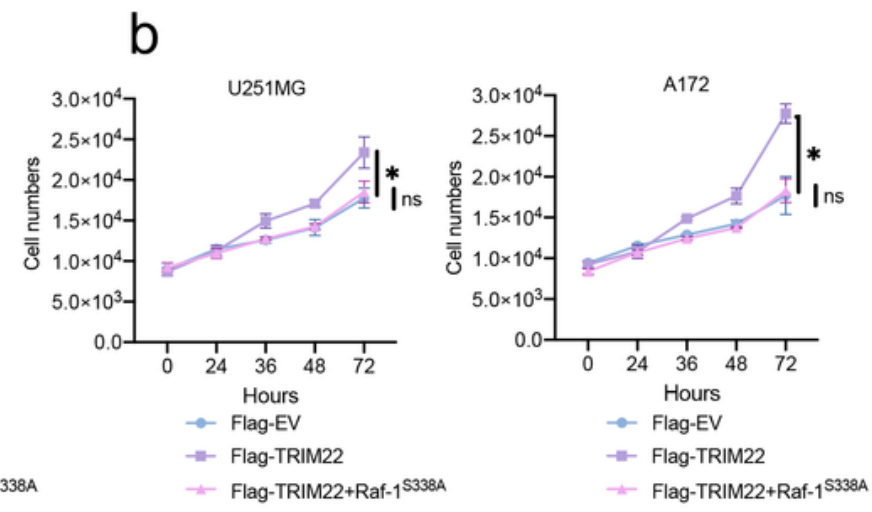
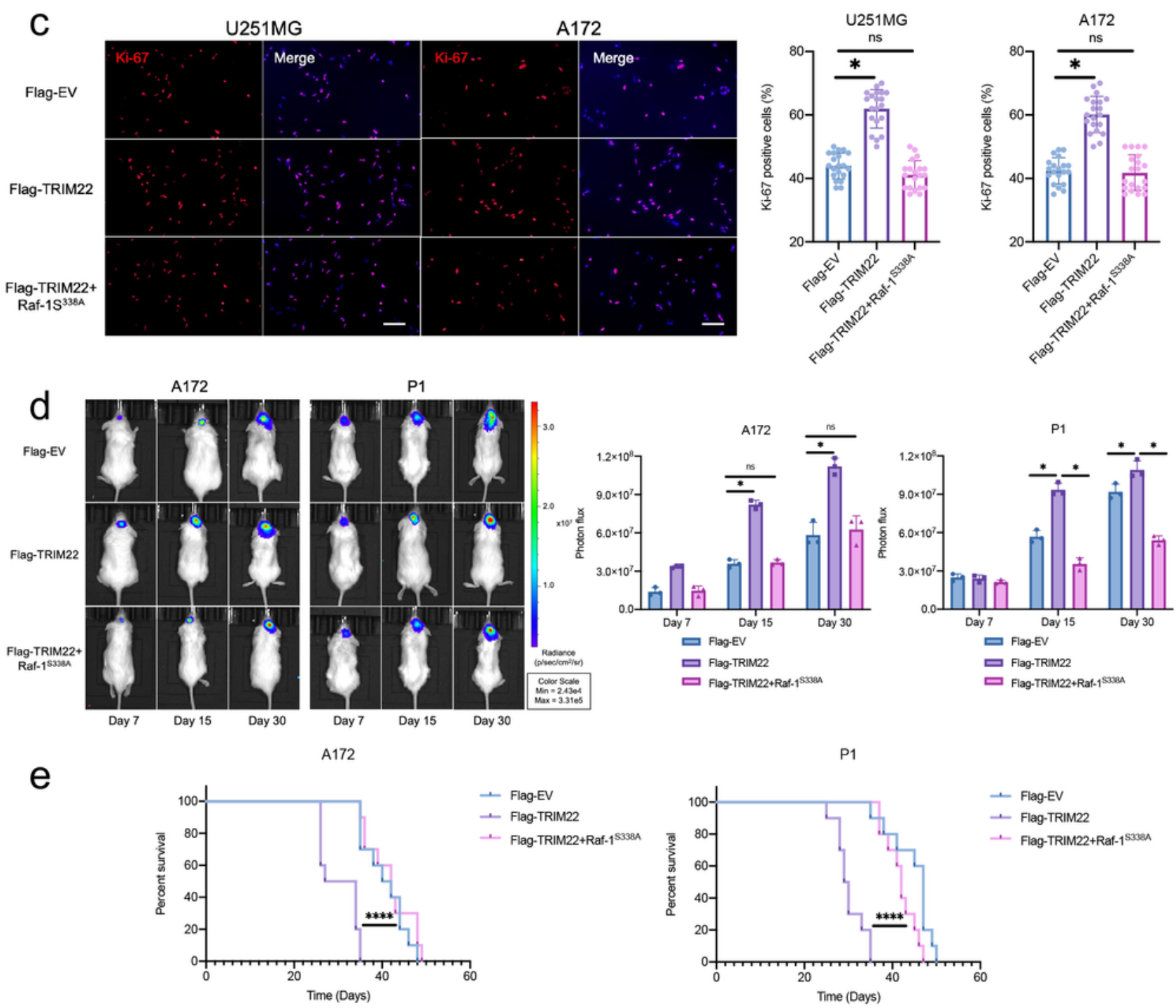

P1

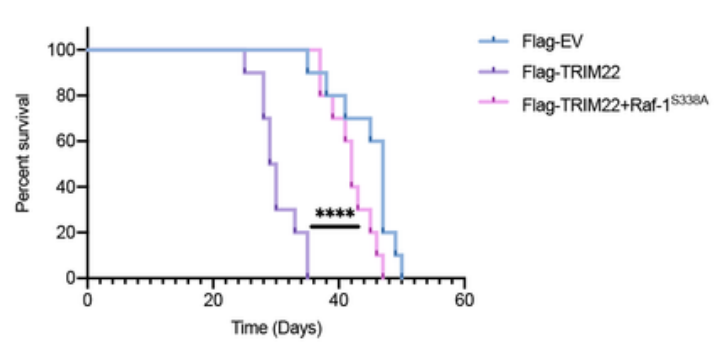

\section{Figure 6}

Raf-1 regulates TRIM22-induced GBM proliferation in vivo and in vitro. a Luciferase activity from U251MG and A172 transfected with Flag-TRIM22 and Raf-1S338A, along with a reporter plasmid carrying the ERK1/2 promoter relative to negative control. b Growth curves generated using cell counting over $72 \mathrm{~h}$ for the cells indicated. c Representative images and quantification of Ki-67 immunofluorescence staining from modified U251MG and A172 cells. Scale bar: $50 \mu \mathrm{m}$. d Representative images and quantification of 
in vivo imaging. e Kaplan-Meier survival analysis and Log-rank test performed with survival data from indicated groups. (All data represent mean \pm SEM $n \geq 3$ ). Student's t test: * $P<0.05$, **** $P<0.0001$.

a

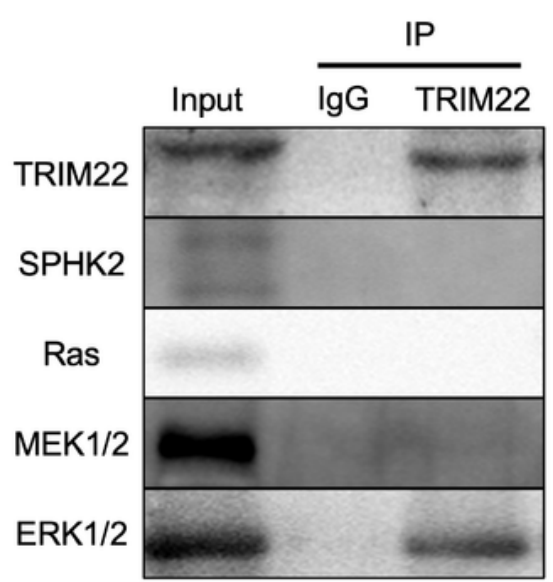

U251MG

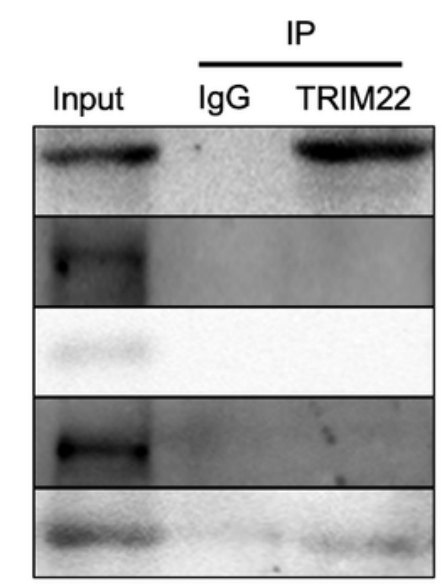

A172

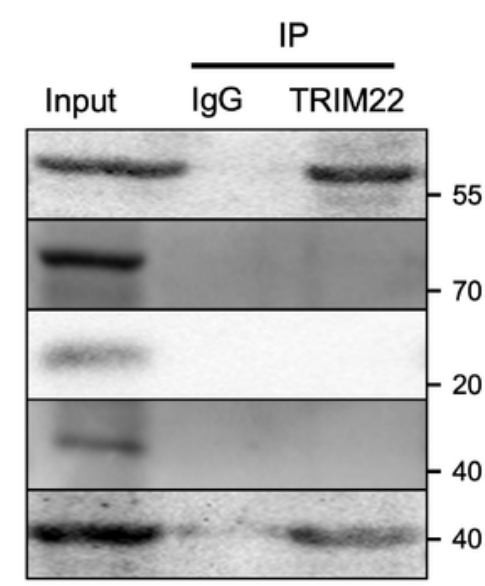

TJ905

b

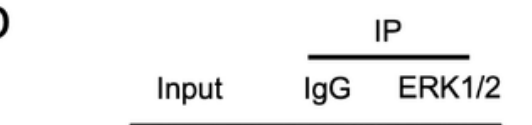

C
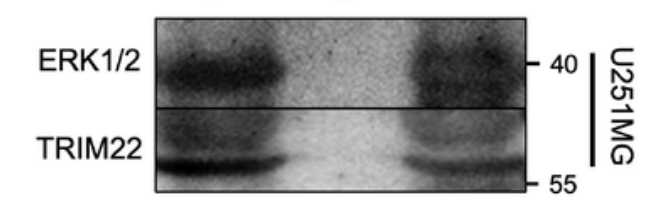

ERK1/2

TRIM22
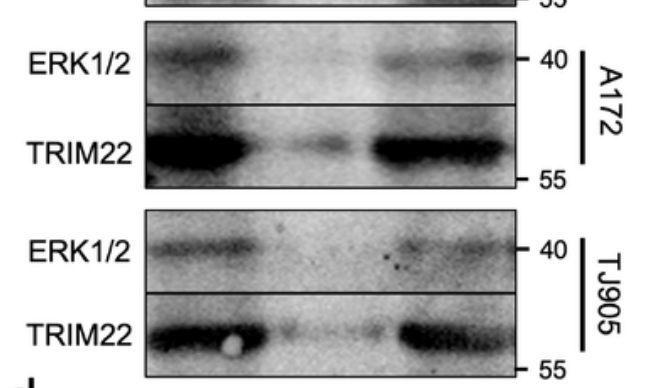

d
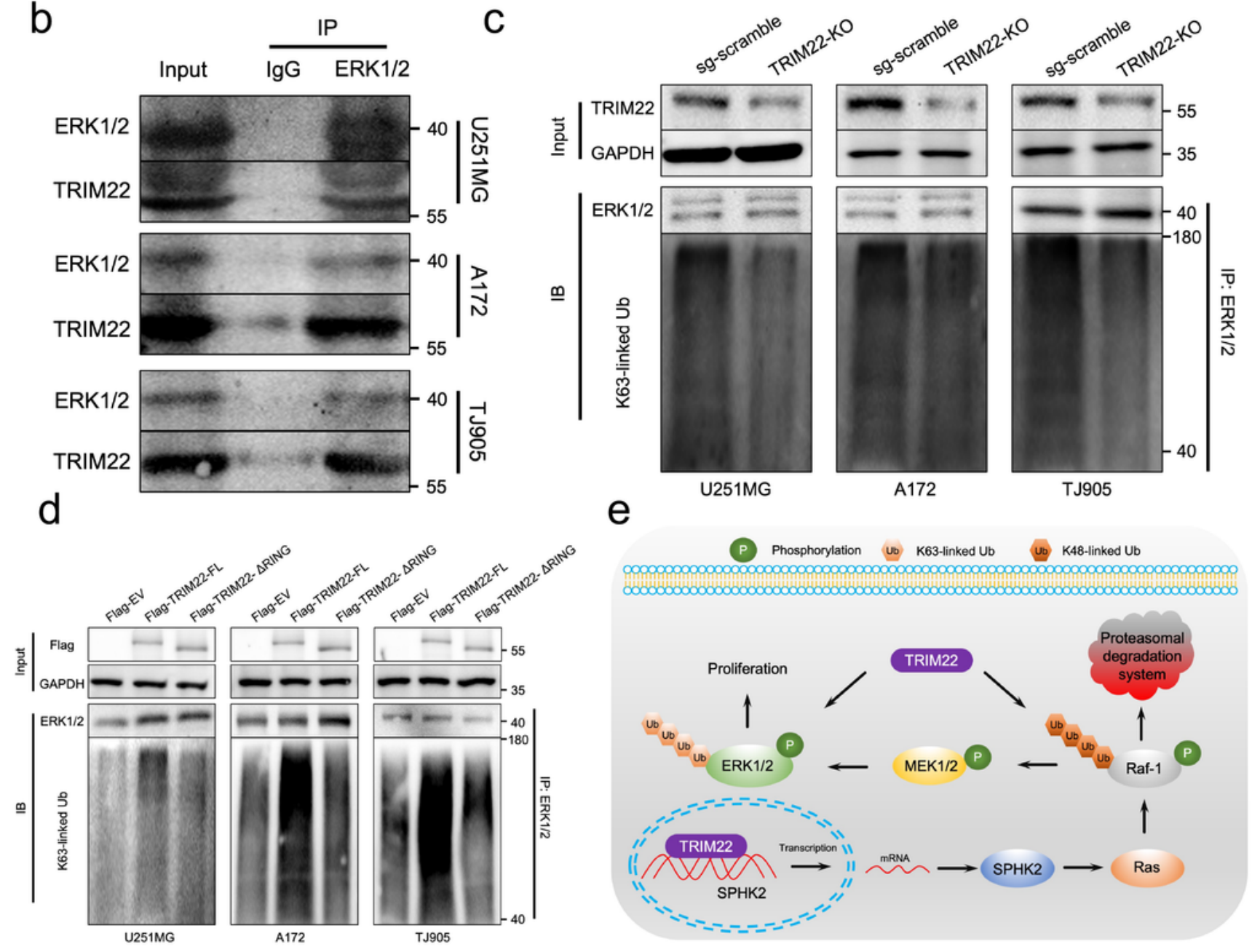

e

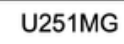

A172

TJ905

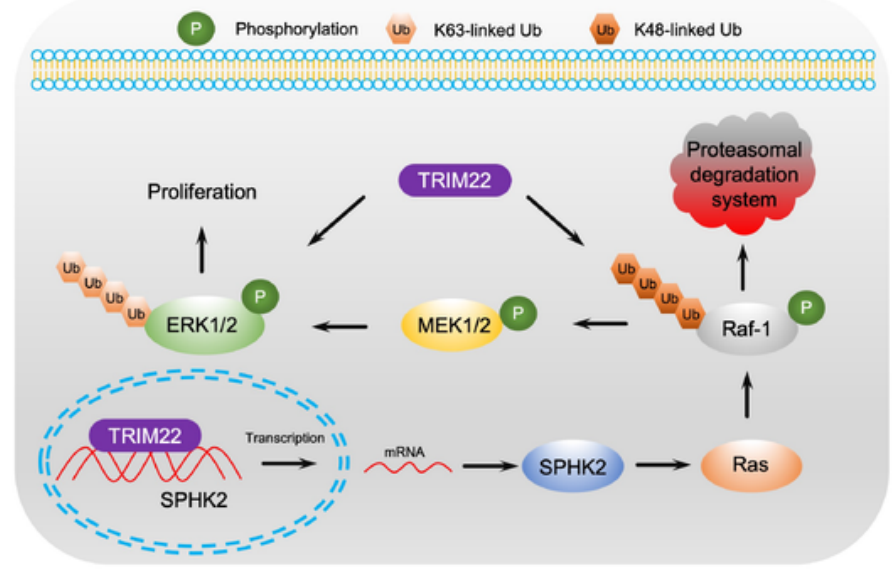

Figure 7

TRIM22 promotes K63-linked ubiquitination of ERK1/2 through its RING domain. a Anti-TRIM22 antibody was used to detect the binding of TRIM22 to SPHK2/MAPK pathway core protein in U251MG, A172 and TJ905. b Association of TRIM22 with ERK1/2 in U251MG, A172 and TJ905. c-d Western blot analysis for 
ERK1/2 of Co-IPs with K63-linkage specific polyubiquitin antibody in the indicated modified U251MG, A172, and TJ905 cells. e graphical model for TRIM22-mediated SPHK2/MAPK activation and GBM growth.

\section{Supplementary Files}

This is a list of supplementary files associated with this preprint. Click to download.

- additionalfile1.docx 